\title{
Charef Mohammed, L'État, le rôle et la place des Marocains du monde dans la région de Souss-Massa
}

Yves Charbit

\section{(2) OpenEdition \\ 1 Journals}

Édition électronique

URL : https://journals.openedition.org/remi/12401

DOI : 10.4000/remi. 12401

ISSN : $1777-5418$

Éditeur

Université de Poitiers

\section{Édition imprimée}

Date de publication : 1 octobre 2019

Pagination : 251-252

ISBN : 979-10-90426-64-1

ISSN : 0765-0752

\section{Référence électronique}

Yves Charbit, "Charef Mohammed, L'État, le rôle et la place des Marocains du monde dans la région de Souss-Massa », Revue européenne des migrations internationales [En ligne], vol. 35 - n 1 et 2 | 2019, mis en ligne le 01 octobre 2019, consulté le 16 avril 2022. URL : http://journals.openedition.org/remi/ 12401 ; DOI : https://doi.org/10.4000/remi.12401 


\section{Notes de lecture}

Charef, Mohammed

L'État, le rôle et la place des Marocains du monde dans la région de Souss-Massa. Rabat : CCME, 2008. $-331 \mathrm{p}$.

ISBN : 978-9954-669-38-9

Le livre se compose de six solides chapitres qui associent réflexion théorique de haut niveau et données empiriques recueillies grâce à un très riche travail de terrain. II faut souligner d'emblée l'importance de ce type d'étude régionale, qui permet de mieux comprendre la réalité profonde des choses, dans la mesure où les migrations s'inscrivent dans une identité régionale très forte.

Le chapitre 1 discute d'abord la relation entre migration et développement. II montre bien la diversité et la complexité des notions en jeu et surtout il pose une question cruciale : le développement permet-il réellement d'arrêter l'immigration? À cet effet, il propose une fine analyse critique du concept de co-développement. Le chapitre 2 consacré aux migrations dans la région Sousse massa " des temps et des lieux " est particulièrement remarquable. II propose une passionnante plongée dans l'histoire pluriséculaire de ce courant migratoire des origines jusqu'à aujourd'hui. II montre la diversité des acteurs, des profils et des destinations, en accordant une attention particulière à l'impact des deux conflits mondiaux sur les flux migratoires.

Le chapitre 3 intitulé avec humour " Migrant marocain et développement ou quand les migrants se mêlent du développement " équilibre les trois niveaux, national régional et local quant à l'impact des migrations. À côté de la présentation des flux de transferts d'argent, il procède à une excellente analyse des conséquences sociales de ces transferts et en particulier les mutations dans I'habitat et dans l'architecture. L'auteur, géographe, s'était révélé historien dans le chapitre 2. Ici le spécialiste de l'urbanisme se fait sociologue. En effet il restait dans ce chapitre à ne pas oublier les acteurs. II nous montre la richesse et la diversité des migrants investisseurs dans le domaine du commerce et dans d'autres secteurs. Ces entrepreneurs ont contribué à " moderniser l'économie régionale " ce dont témoigne une " success story ".

Le chapitre 4 consacré aux associations de migrants pose le problème de leur rôle dans le développement régional. II s'ouvre par une riche réflexion sur la participation citoyenne et la démocratie représentative au Maroc. Ce chapitre montre en particulier le dynamisme du monde associatif dans la région par rapport aux autres (voir le spectaculaire graphique 6 en page 187). Dans le cas du Douar Ouled Mimoun, ce douar " presque ordinaire ", la vie a été profondément changée par les migrants et leur association créée en 1994.

Le chapitre 5 s'attache à d'autres associations particulièrement importantes par le rayonnement et par leur action. La première est Migration et développement créé en 1986 et qui a une approche en quelque sorte horizontale du développement. Elle se préoccupe du genre, de l'environnement, de la gouvernance locale, des activités génératrices de revenus, d'éducation et même de formation à la gestion associative. Dans le cas de la commune rurale de Arbaa Sahel, le témoignage du président de la commune rurale, Brahim Safini, met en valeur le rôle des émigrés de la diaspora économique. Enfin, le chapitre souligne les apports des associations des Marocains résidents à l'étranger et leur perception positive par les acteurs locaux. 
Le chapitre 6 s'attache à la question de la territorialisation et, à travers elle, à une réflexion sur la politique locale et régionale : en quoi et comment les structures étatiques mobilisent-elles les Marocains résidant à l'étranger dans le développement local ? Sont décrits les rôles du Centre régional d'investissement de la région, de la Chambre de commerce d'industrie de services, de l'Agence de développement social de la région, de l'Agence nationale de la promotion de l'emploi et des compétences. Tout ceci conduit l'auteur à faire des propositions concrètes notamment à propos de Taroudant et de Tiznit.

La conclusion de l'ouvrage interroge à juste titre sur la complexité des liens actuels, qui dépassent la seule question des transferts d'argent. Elle insiste sur les risques liés à la politique migratoire des pays d'installation, qui pourrait aboutir à une fragilisation des communautés marocaines vivant l'étranger. Aussi l'auteur plaide-t-il pour le renforcement des liens avec la société d'origine alors que les migrants de la première et deuxième génération montrent peu d'empressement dans I'investissement productif. À ses yeux l'un des enjeux majeurs du développement durable au Maroc est d'arriver à utiliser au mieux les capacités des associations de migrants. Le bilan est certes positif et l'implication des MRE dans développement local a été indéniable qu'il s'agisse de l'électrification, de l'abduction d'eau, de l'éducation, de la santé, voire du renforcement de l'identité locale. Le livre se termine par quelques recommandations qui sont en réalité de véritables propositions d'une politique du développement local qui se donnerait les moyens d'associer les migrants.

Ce rapide survol de cet ouvrage aura montré son extraordinaire richesse et son authentique dimension interdisciplinaire, car il associe géographie humaine, économie, histoire, science politique, ethnographie. II faut souligner le brio avec lequel l'auteur parvient à mobiliser de manière extraordinairement convaincante les échelles spatiales (du niveau local au régional et au national), les échelles temporelles (court terme, moyen terme et long terme) et enfin les échelles sociales, puisque l'analyse du migrant n'est jamais dissociée de sa famille, de sa communauté d'appartenance des associations auxquelles il participe, et bien évidemment de la dialectique entre les acteurs individuels, la société civile et les institutions politiques, que ce soit l'État ou la région. Ce livre important sera sans doute une référence pour la connaissance de cet enjeu majeur qu'est le développement local et il est à souhaiter que des études comparables soient réalisées sur d'autres régions afin d'avoir une vue d'ensemble de la relation entre migration et développement au Maroc.

Yves Charbit

Démographe, Professeur émérite CEPED, Université Paris Descartes/IRD

\section{Séraphin, Gilles (éd.)}

Famille et migration, Recherches familiales, 13. - Paris : Union Nationale des Associations Familiales, 2016. - 145 p.

La migration, même lorsqu'elle ne concerne qu'un membre de la famille, « marque tout autant le vécu de l'ensemble de la famille " (p. 3). Gilles Séraphin, par ces mots, insiste sur l'importance de s'intéresser aux acteurs de la migration, migrants et non migrants et notamment à la famille où chaque membre voit sa place évoluer par la migration par une " restructuration familiale au moins sur le plan symbolique " (p. 3). Et à l'instar d'ouvrages récents (Imbert et al., 2018 ; Kraler et al., 2011), la famille est au cœur de l'attention de plusieurs auteurs réunis dans ce dossier thématique " Famille et Migration ". Pour ce treizième numéro de Recherches familiales, I'introduction de Gilles Séraphin pose les bases de la réflexion sur la relation entre famille et 
migration qui sera le fil conducteur des six articles francophones. Le dossier est pensé en trois parties énoncées dans la partie introductive : " La migration dans le roman familial : la construction de l'identité ", " Migration et relations familiales " et " Migration, droit et institution ».

La première partie aborde ainsi I'histoire et l'identité familiale forgée à travers la migration et surtout son énoncé après la migration. Cette partie aborde notamment les questions d'identité que se posent les descendants de migrants. Ainsi, le premier article d'Anne Tatu-Colasseau, aborde la transmission de la mémoire et de la culture dans des familles maghrébines et son rôle dans l'intégration sociale des enfants. Les deux sont liés ici, car " pour se soumettre à l'injonction de réussite sociale, les descendants ont besoin de garder les "pieds trempés" dans les références d'origine, invalidant l'hypothèse d'une assimilation par la rupture culturelle » (p. 20). AnneTatuColasseau conclut son article en soulignant l'importance des transmissions féminines. La mère " se révèle actrice pivot des transmissions décisives " (p. 20) et les filles seront plus réceptives à ces transmissions culturelles et mémorielles, moteur pour elles de leurs projets d'ascension sociale. Ce lien parents-enfants est poursuivi dans I'article suivant d'Elsa Lagier. La sociologue lie le roman familial avec l'intérêt et l'engagement politique des enfants d'immigrés et constate que " le parcours migratoire des parents influence la socialisation politique de leurs enfants à partir du moment où l'histoire personnelle familiale des parcours concrets se trouve réinscrite dans une histoire plus collective qui lui donne du sens " (p. 33). Les enfants mobilisent, consciemment ou non, cette histoire familiale dans leur rapport à la politique et donnent un sens à leurs situations à travers cette histoire.

La deuxième partie se focalise sur les relations familiales au sein de la migration. Gilles Séraphin évoque la double absence de l'émigré qui modifie les relations familiales, avec une diversité de situations aujourd'hui qui peuvent affecter les relations intergénérationnelles entre enfants partis et parents restés. Deux articles évoquent ces questions de génération et de distance au sein de la famille. Ainsi, le texte de Carole Beaugendre, Didier Breton et Claude-Valentin Marie donne l'exemple de migrations d'outre-mer à la métropole et évoque une des conséquences du départ sur les populations restées. En effet, à partir d'un travail sur I'Enquête Migration, Famille, Vieillissement (2009-2010) et des données du recensement, les trois auteurs montrent " l'importance de l'éclatement des familles des natifs des DOM " (p. 51) avec le départ des plus jeunes. Face à cet éclatement, l'enjeu principal est la prise en charge des personnes âgées dans des territoires où le vieillissement est important et où le nombre " d'enfants-aidants " tend à diminuer par la migration et la baisse de la fécondité. L'inquiétude est d'autant plus forte que les auteurs rappellent que " les DOM sont particulièrement déficitaires en équipement d'accueil de ce public " âgé. Mathilde Plard poursuit le thème de l'intergénérationnel avec l'article suivant. Elle explique comment la décohabitation des enfants et parents crée une nouvelle organisation dans les relations de care et donc une prise en charge à distance pour des familles transnationales brahmanes. Ces enjeux de prise en charge des populations âgées au pays d'origine ont d'ailleurs été rappelés dans un ouvrage récent (Nowik et Lecestre-Rollier, 2015) où Mathilde Plard évoque également le cas des familles transnationales indiennes, mais où était aussi évoquée la situation de Mayotte dont on peut faire des rapprochements avec celle des Antilles ou de La Réunion.

Enfin les deux derniers textes sont de nature plus juridique et institutionnelle et abordent la famille au cœur du droit et des institutions françaises. Le premier, d'Anne Wyvekens se base sur une enquête faite dans deux tribunaux de grande instance et place le droit familial en contexte de 
migration. L'auteure montre comment la justice familiale face à des justiciables immigrés ou d'origine immigrée met en scène une double appartenance produite par la migration plutôt que des " conflits de normes " qui opposeraient « le droit du pays d'accueil et les systèmes de valeurs de populations venues d'ailleurs " (p. 76). Pour les magistrats, l'examen individuel doit se faire de manière pragmatique face à la dimension collective qu'est l'immigration, ils " voient les individus, leur réalité immédiate [...] avant ce qu'ils représentent socialement " (p. 77). Enfin le dernier article de Myriam Hachimi Alaoui, évoque les politiques publiques d'intégration destinées à " inculquer un socle de "valeurs" aux "nouveaux entrants" dans la communauté politique "(p. 92). Elle revient sur les différentes étapes de cette réglementation de l'immigration familiale et met la focale sur les « femmes d'immigrées " vue comme " pivot de l'intégration des familles " (p. 83). En parallèle des discours autour de la mise en place de cette politique d'intégration, elle dresse le portrait des dispositifs pour intégrer les familles migrantes, notamment des formations civiques qui y sont associées. Face à des "valeurs " souvent déconnectées de la vie de ces migrants, les tentatives pour les " inculquer " semblent vaines, voire parfois contreproductives.

Nous pouvons regretter le manque d'éléments conclusifs et liants pour clore ce dossier et développer une dimension plus globale sur les rapports familiaux en migration. Cependant, la partie introductive de Gilles Séraphin a apporté en amont quelques éléments de réflexion sur l'évolution familiale au cœur des migrations, tout en résumant parfaitement les différents articles. Dans tous les cas, le dossier remplit l'objectif $d$ '« étudier ce que la migration d'une unité familiale ou de l'un de ses membres [...] construit comme pratiques familiales " (p. 7) et plus largement comme mémoire et identité familiales et sociales. Les différentes études de cas permettent d'enrichir la réflexion autour d'un champ d'études des migrations encore en construction.

\section{Références bibliographiques}

Imbert Christophe, Lelièvre Éva et Lessault David (2018) La famille à distance : mobilités, territoires et liens familiaux, Paris, INED, $376 \mathrm{p}$.

Kraler Albert, Kofman Éléonore, Kohli Martin and Schmoll Camille (2011) Gender, Generations and the Family in International Migration, Amsterdam, Amsterdam University Press, 404 p.

Nowik Laurent et Lecestre-Rollier Béatrice (2015) Vieillir dans les pays du sud. Les solidarités familiales à l'épreuve du vieillissement, Paris, Karthala, 312 p.

Jordan Pinel

Géographe, Doctorant Migrinter/Université de Poitiers

\section{Arab, Chadia}

Dames de fraises, doigts de fée, les invisibles de la migration saisonnière marocaine en Espagne. - Casablanca : En toutes lettres, 2018. - 185 p.

ISBN : 978-9954-987-90-2

Chadia Arab (Géographe, CNRS-ESO) rend compte du phénomène de migration circulaire de femmes marocaines entre le Maroc et l'Espagne en mobilisant différentes enquêtes de terrain réalisées, principalement des interviews des acteurs concernés par ce phénomène, entre 2008 et 2017.

Le style de rédaction tranche avec des écrits académiques classiques - à mettre en lien avec le format usité par la maison d'édition choisie - puisqu'elle nous propose une ethnographie sous la forme d'investigation permettant un accès au plus grand nombre.

L'ouvrage est divisé en six chapitres abordant à la fois l'aspect institutionnel 
de ce type de migration, et le parcours des femmes actrices principales. L'auteure montre tout d'abord quels types de femmes sont concernés, et les changements que cette migration a amenés tant dans leur gestion du quotidien, que dans leur perception de I'avenir. Ensuite elle indique comment ce programme de migration circulaire a été mis en place dans le but de lutter contre la migration illégale, tout en annonçant de manière concise les méfaits de cette migration institutionnalisée, sur ces femmes.

Chadia Arab suit une méthodologie aussi riche que rigoureuse puisqu'elle procède principalement à des interviews et immersions et non uniquement à des observations espacées dans le temps, pour étudier ce phénomène.

À l'aide de multiples témoignages, l'auteure objective ce nouveau de type de migration institutionnalisée dite " circulaire " permettant à certaines femmes marocaines de migrer pour travailler en Espagne.

L'auteure explicite au fur et à mesure des chapitres comment ces dernières ne furent pas passives, mais pleinement actrices dans le processus, afin de tenter d'améliorer leurs conditions de vie quotidienne, mais également celles des familles restées au Maroc.

Dans un premier chapitre, Chadia Arab explicite dans quelle mesure la migration de femmes marocaines à Huelva fut une opportunité pour sortir des grandes difficultés sociales et économiques, tout en n'omettant pas de rendre compte des méfaits qu'implique cette mobilité. Cette province située au sud de l'Espagne est surnommée "la province de l'or rouge ", pour évoquer les fraises. Chaque année, des coopératives espagnoles embauchent des milliers de femmes, majoritairement d'Europe de l'Est (Pologne, Roumanie) et du Maghreb (Maroc) pour planter et cueillir les fraises, moteur économique de cette région.
La division sociosexuée du travail est au cœur du phénomène circulatoire, car ne concerne que des femmes embauchées pour leur minutie, et leurs " doigts de fées ॥.

L'État espagnol observe de son côté, cette migration comme une opportunité face à la diminution de la population dans cette région, amenant une baisse de maind'œuvre féminine peu qualifiée.

Le programme de " gestion éthique de l'immigration saisonnière " matérialise la migration circulaire et consiste à faire venir des femmes marocaines quelques mois en Espagne en leur octroyant des visas prévus à cet effet, puis à les renvoyer chez elles le reste de l'année.

Selon l'auteure, ce programme a pour but de pallier les besoins économiques espagnols, de contrôler la migration vers I'Union européenne, mais également de développer les pays d'origine des femmes grâce aux flux de devises. Appartenant au programme européen AENEAS (Programme d'assistance technique et financière à des pays tiers dans le domaine de l'émigration et de l'asile) de I'UE, ce programme de migration a pour but de lutter contre la migration clandestine.

Le Maroc ayant des relations étroites avec I'UE, dans le cadre de ces migrations circulaires, il a avec l'Espagne créé deux institutions clés : I'ANAPEC embauchant les femmes au Maroc selon les demandes des coopératives et organes de centralisation des informations, et la FUTEH en liaison avec la Mairie de Cartaya (en Espagne), organisme d'accompagnement. Ces organismes ont été mis en place dans un but de transparence du système, créant des étapes institutionnalisées entre l'embauche au Maroc et le travail en Espagne. Ce type de migration est donc contrôlé : toutes les femmes ne peuvent pas être éligibles au contraire. 
L'auteure avance avec précision les différentes conditions préalables à cette circulation : femmes marocaines rurales puisque considérées comme plus dociles. Cette circulation est encadrée tant par l'État de départ que par l'État d'arrivée : chaque étape de la migration encadrée par I'ANAPEC au Maroc puis la FUTEH en Espagne.

Ce type de migration se signale pour son aspect temporaire et incertain. L'employeur a droit de regard sur la fin du contrat de travail et l'expulsion de la travailleuse.

Une des forces de ce travail est de toujours considérer l'imbrication des rapports de pouvoir comme organisateur central de la circulation des femmes concernées ; grâce notamment aux données de la municipalité de Cartaya.

Les femmes s'inscrivant à ce type de migration sont issues de milieux paupérisés de la société marocaine et plus particulièrement ruraux. Toutes les concernées sont âgées entre trente et cinquante ans, ont des enfants à charge, parfois analphabétisées.

Chadia Arab rappelle avec justesse que les femmes choisies pour la réussite de ces programmes sont des femmes qui n'avaient jamais quitté leur village. Cela facilite leur renvoi après les récoltes en pariant également de leur docilité.

Ces départs ne se font pas sans difficulté puisque l'auteure narre les difficultés initiales rencontrées par ces femmes. Cela concerne la somme d'argent nécessaire pour compléter leurs dossiers administratifs qui mène à un endettement, et les négociations avec les familles pour l'autorisation de partir à l'étranger seules.

Malgré cela, questionnaire à I'appui, Chadia Arab explique que les candidates trouvent un intérêt humain à avoir quitté le Maroc, et ce malgré des conditions de travail plus que difficiles.
Cependant il faut prendre en compte la subjectivité des réponses aux questionnaires qui peuvent alors être erronées, car ces femmes subissent une certaine pression de leur employeur, et du système lui-même, car redoutent de ne plus être rappelées.

D'un point de vue économique, l'auteure remarque que ces dames de fraises par leur rémunération et expérience en Espagne sont plus autonomes et libres. L'autonomie financière en est la preuve la plus marquante, car leur permet d'acquérir un certain pouvoir décisionnel dans la famille. Cette migration leur permet d'être plus mobile une fois au Maroc, et plus à l'aise, lorsqu'entourées d'hommes. Elles passent d'un rôle plus traditionnel féminin (lié à l'injonction à la maternité) au rôle de cheffes de famille et actrices économiques. En outre, ces femmes marocaines choisies pour leur précarité ont prouvé leur force face au travail et à l'éloignement, on parle de « zoufria » (femmes capables).

Cependant, cet ouvrage n'omet pas les aspects plus négatifs. La plupart retrouvent leur rôle de "femme " au sein de la famille, la division sexuelle du travail se recompose dès qu'elles rentrent chez elles. Cette soumission est même présentée comme institutionnalisée, car elles nécessitent un accord masculin pour participer à ces programmes de migration. Le choix de migrer est potentiellement rarement personnel, mais répond aux besoins des familles.

Cette " émancipation " est donc réelle, mais temporaire. L'image renvoyée de ces femmes au Maroc est loin d'être aussi positive, en effet on les assimile à de mauvaises mères, voire déstabilisatrices, de la valeur familiale, en se voyant accusées de prostitution en Espagne.

Enfin, dans les deux derniers chapitres Chadia Arab explique les effets pervers de cette immigration circulaire ; puisque 
la frontière entre celle-ci et l'immigration clandestine est ténue.

Loin d'être un processus gagnantgagnant comme imaginé de jure, ce dernier est un moyen d'éviter toute volonté d'installation de ces femmes en Espagne ; au risque de devenir " sans-papières ".

L'auteure s'efforce donc à mettre en avant les répercussions sociales de ce phénomène économique. Cette migration institutionnalisée est fortement critiquée négativement par des organismes agissant pour les droits humains au Maroc. Ils dénoncent l'exploitation de ces femmes précaires et l'atteinte aux droits humains (comme l'attente au droit de circulation).

L'auteure explicite que ce système institutionnalisé sous le regard de l'Union européenne est à même de créer des Harragas (migrante.es clandestin.e.s). En n'employant les femmes que sur des périodes courtes, les employeurs peuvent diminuer les effectifs des coopératives d'une année à l'autre. Ceci impacte la précarité de ces femmes qui, n'ayant pas les moyens de reconstituer leurs dossiers chaque année, préfèrent rester sur place malgré l'interdiction juridique. Ce phénomène est paradoxal, car allant à l'encontre de l'esprit de la migration circulaire. Ces dernières se retrouvent dans des situations de contraintes concrètes. Elles tentent d'acheter leurs contrats de travail pour parvenir à la régularisation tandis que d'autres cherchent une protection masculine en tentant de contracter mariage, bien souvent sans succès.

Chadia Arab conclut son ouvrage par un ultime chapitre au titre évocateur " une immigration jetable ". Loin de créer une migration sociale et éthique, I'Union européenne et le Maroc ont mis en place un système de " migration jetable " où les femmes sont sollicitées seulement si besoin, et en fonction des conditions économiques espagnoles. Les employeurs peuvent librement choisir leurs effectifs.
Ce processus de migration circulaire fut établi dans le seul but de pallier le besoin économique des deux pays concernés et de lutter contre l'immigration clandestine. Les conditions de vie de ces femmes et leurs précarités ne sont utilisées qu'à des fins économiques et politiques. Cependant, et malgré des conditions de travail et vie indignes, l'ouvrage visibilise au mieux les espaces interstitiels de liberté et le certain bénéfice social et économique que ces femmes retirent de ces emplois saisonniers.

\section{Nouri Rupert Sociologue \\ LCSP-CEDREF/Université Paris Diderot}

Arab, Chadia

Dames de fraises, doigts de fée, les invisibles de la migration saisonnière marocaine en Espagne. - Casablanca : En toutes lettres, 2018. - 185 p.

ISBN : 978-9954-987-90-2

Nous sommes tou.t.es des \#DamesDeFraises, Mediapart: Le blog de Chadia Arab, 2018. [en ligne]. URL : https://blogs.mediapart.fr/ chadia-arab/blog/130618/nous-sommes-toute-s-des-damesdefraises

Entre 2008 et 2010 les autorités consulaires commerciales espagnoles et marocaines développèrent l'idée de réglementer une migration circulaire entre les deux nations pour les saisonnières de récoltes agricoles des immenses cultures de fraises de la Huelva, dans l'Ouest andalou, proche de l'Algrave portugais : ce programme éthique mis au point devait éviter les aventures des sans-papiers et les diverses formes de leurs exploitations qui ponctuent les conflits entre travailleurs-euses marocain'e-s et propriétaires agricoles à lest de l'Andalousie, dans la province d'Almeria-El Ejido, dans la " mer des serres". Deux structures furent concernées : au Maroc, le ministère de I'Emploi confia à I'ANAPEC (Agence Nationale de promotion de l'emploi et des compétences) la charge d'accueillir les candidates aux activités 
agricoles saisonnières, de les orienter et de les accompagner. En Espagne c'est la FUTEH (Fundacion para trabajadores de estranjeros en Huelva) localisée dans la mairie de Cartaya qui réalisait l'accueil et la répartition des "doigts de fées ", surnom des cueilleuses proposé par Chadia Arab recueillant une unanimité de témoignages sur leur délicatesse dans la cueillette des fraises.

II s'agissait d'une régulation des mouvements saisonniers qui devait maintenir ces femmes dans un statut permanent de migrantes circulaires, enrichissant les familles restées au Maroc, et évitant dans le Sud espagnol les immigrations définitives liées au droit du travail des travailleurs temporaires.

C'était oublier et, de fait, renforcer, les processus de domination auxquels les femmes marocaines étaient soumises : célibataires avec enfants, divorcées en charge de famille, mariées avec des époux sans travail, étaient nombreuses parmi ces Marocaines de trente-cinq à quarantecinq ans astreintes à un travail méticuleux de cueillettes en plusieurs passages et accroupies dans I'humidité de l'arrosage constant et la chaleur des champs sans arbres. Dominations de genre, au Maroc, où le statut de la femme rurale est défini par toutes sortes de soumissions, et en Espagne, embauchées par des hommes, les propriétaires, et constamment surveillées par des contremaîtres espagnols, pendant leur travail et durant leurs pauses et leur vie nocturne dans des hangars-dortoirs. Cette migration genrée en situation de dépendance économique totale voyait se démultiplier les rapports de domination masculine. Chadia Arab signale à quel point le " ni d'ici ni de là-bas " de l'immigrant, formulé en 1999 par Abdelmalek Sayad prend une forme paroxystique... avec une singulière exception dans un des premiers portraits de transmigrantes circulaires présenté comme emblématique : elle décrit la réussite d'une jeune mère enrichissant sa famille au Maroc par des achats productifs de terres et de biens ; nous sommes typiquement dans un cas opposé à ceux présentés par Sayad, et développé plutôt par Lamia Missaoui en 1995, « le migrant et d'ici et de là-bas et de I'entre deux ", riche analyse triadique qui contribuera à sa définition de "l'étranger de l'intérieur ". L'« entre deux ", parcours social, économique, moral, transnational, malgré la cohérence de son montage politico-administratif, opère comme un incitateur à la dérogation, à la relative libération de leur condition par ces jeunes femmes: la proximité d'autres transmigrants n'y est pas pour rien, femmes polonaises qui se réclament de leur européanité pour diverses revendications, hommes subsahariens, qui ont pris de l'ascendance sur leurs patrons et courtisent les Marocaines, etc.

Chadia Arab nous introduit finement, anthropologiquement (la danse des " alters "), dans la première partie de son ouvrage, aux histoires de vie liées à ces cheminements transnationaux puis elle nous livre (pp. 85-106) une enquête sociographique méticuleuse sur les origines, les localisations, les contours, fratries, revenus familiaux, etc. qui complète l'enquête par proximité, par accompagnement, et permet de situer les enjeux sociétaux de cette migration circulaire de femmes marocaines.

II est rare de lire une recherche de terrain aussi sensible et précise, nouant sans arrêt un continuum entre trajectoires individuelles et collectives, voguant du plus sensible, empathique, rapport aux personnes aux plus distantes récollections collectives de l'enquête intensive.

L'information contextuelle concerne surtout le Maroc. II faut resituer cette recherche dans une suite de travaux concernant le Levant espagnol, de la frontière du Perthus à I'Ouest andalou. Des enquêtes, en collaboration, de David Sempere, Oriol Romani, Lamia Missaoui, Alain Tarrius de 1999, ont mis en évidence les modalités 
de développement de réseaux marocains le long du Levant ibérique, incluant la " traite des travailleurs saisonniers " et les premières révoltes des Marocains d'El Ejido travaillant sous serre, celles de Swanie Potot, 2003, concernent les travailleuses roumaines dans les serres andalouses, celles encore de Fatima Lahbabi et Pilar Rodriguez Martinez (2002-2004) sur la prostitution massive de jeunes Marocaines en Andalousie, et enfin celles d'Olivier Bernet et d'Alain Tarrius sur les trafics de femmes balkaniques vers le Levant espagnol (2011-2015).

L'expérience relatée par Chadia Arab se voulait « réparatrice » des tensions révélées par les précédentes études. La chercheure montre l'inanité des figurations de maîtrise administrative des frontières politiques: d'autres frontières, morales, s'imposent rapidement, générées entre autres par la rigidité administrative des montages internationaux, ignorants des désirs, aspirations, soumissions et consciences de ces phénomènes permis par l'entre-deux transmigratoire...

\section{Références bibliographiques}

Lahbabi Fatima y Rodriguez Martinez Pilar (2005) Migrantes y trabajadoras del sexo, Del Blanco, $214 \mathrm{p}$.

Missaoui Lamia (1995) Généralisation du commerce transfrontalier : petit ici, notable là-bas, Revue Européenne des Migrations Internationales, 11 (1), pp. 53-75.

Missaoui Lamia, Sempere Juan David et Oriol Romani (2000) Apparition des comptoirs, et des réseaux souterrains marchands, marocains le long du Levant Ibérique, Rapport de recherche DG12 Europe (5ème PCRD), $132 \mathrm{p}$.

Sayad Abdelmalek (1999) La double absence. Des illusions de l'émigré aux souffrances de l'immigré, Paris, Seuil, $437 \mathrm{p}$.

Tarrius Alain (2014) Transmigrations européennes de travailleuses du sexe balkaniques et caucasiennes accompagnées de parentèles féminines, Revue Tiers Monde, 217 (1), pp. 25-43.
Tanius Alain (2002) La mondialisation par le bas. Les nouveaux nomades de l'économie souterraine, Paris, Balland, $175 \mathrm{p}$.

Péraldi Michel (Éd.) (2001) Cabas et containers. Activités marchandes informelles et réseaux migrants transfrontaliers, Paris, Maisonneuve et Larose, $361 \mathrm{p}$.

Potot Swanie (2005) La place des femmes dans les réseaux migrants roumains, Revue Européenne des Migrations Internationales, 21 (1), pp. 243-257.

Alain Tarrius Sociologue et anthropologue, Professeur émérite LISST, Université de Toulouse le Mirail/CNRS 